\title{
FSHR-1/GPCR Regulates the Mitochondrial Unfolded Protein Response in Caenorhabditis elegans
}

\author{
Sungjin Kim* and Derek Sieburth*,t,1 \\ ${ }^{\star}$ Zilkha Neurogenetic Institute, Keck School of Medicine and ${ }^{\dagger}$ Department of Physiology and Neuroscience, University of Southern \\ California, Los Angeles, California 90033
}

\begin{abstract}
The mitochondrial unfolded protein response (UPR $\left.{ }^{m t}\right)$ is an evolutionarily conserved adaptive response that functions to maintain mitochondrial homeostasis following mitochondrial damage. In Caenorhabditis elegans, the nervous system plays a central role in responding to mitochondrial stress by releasing endocrine signals that act upon distal tissues to activate the UPR ${ }^{m t}$. The mechanisms by which mitochondrial stress is sensed by neurons and transmitted to distal tissues are not fully understood. Here, we identify a role for the conserved follicle-stimulating hormone $\mathrm{G}$ protein-coupled receptor, FSHR-1, in promoting UPR ${ }^{m t}$ activation. Genetic deficiency of fshr- 1 severely attenuates UPR ${ }^{m t}$ activation and organism-wide survival in response to mitochondrial stress. FSHR-1 functions in a common genetic pathway with SPHK-1/sphingosine kinase to promote UPR ${ }^{m t}$ activation, and FSHR-1 regulates the mitochondrial association of SPHK-1 in the intestine. Through tissue-specific rescue assays, we show that FSHR-1 functions in neurons to activate the UPR ${ }^{m t}$, to promote mitochondrial association of SPHK-1 in the intestine, and to promote organism-wide survival in response to mitochondrial stress. We propose that FSHR-1 functions cell nonautonomously in neurons to activate UPRmt upstream of SPHK-1 signaling in the intestine.
\end{abstract}

KEYWORDS FSHR-1; paraquat; sphingosine kinase; UPR mt

T HE mitochondrial unfolded protein response (UPR ${ }^{\mathrm{mt}}$ ) functions to maintain mitochondrial protein homeostasis in response to mitochondrial dysfunction caused by mitochondrial DNA damage, incorrect mitochondrial protein folding, or impaired oxidative phosphorylation. Failure to appropriately control and maintain protein homeostasis in the mitochondria is associated with the development of numerous diseases, neurodegeneration, and ageing (Durieux et al. 2011; Liu et al. 2014; Pellegrino et al. 2014; Nargund et al. 2015; Fiorese et al. 2016; Martinez et al. 2017). The $\mathrm{UPR}^{\mathrm{mt}}$ is initiated when mitochondrial proteostasis is disrupted, the detection of which by mitochondrial and cytosolic factors leads to epigenetic modifications and transcriptional responses in the nucleus that restore mitochondrial function. A critical sensor and activator of the UPR ${ }^{\mathrm{mt}}$ in Caenorhabditis elegans and in mammals is the leucine zipper transcription factor ATSF-1/ATF5 (Fiorese et al. 2016). ATFS-1 is normally

Copyright @ 2020 by the Genetics Society of America

doi: https://doi.org/10.1534/genetics.119.302947

Manuscript received October 2, 2019; accepted for publication December 2, 2019 . published Early Online December 4, 2019.

${ }^{1}$ Corresponding author: Zilkha Neurogenetic Institute, University of Southern California, 1501 San Pablo St., Los Angeles, CA 90033. E-mail: sieburth@usc. edu targeted to mitochondria where it is degraded, but upon mitochondrial stress, mitochondrial import is disrupted and ATFS-1 is targeted instead to the nucleus where it regulates the expression of a cascade of genes including the conserved HSP70-like chaperone, $h s p-6$, which is targeted to the mitochondria to restore protein folding (Nargund et al. 2012). The intestinal UPR ${ }^{\mathrm{mt}}$ can be activated by mitochondrial stress originating either cell autonomously in the intestine or cell nonautonomously in the nervous system. Mitochondrial stress in neurons activates the UPR ${ }^{\mathrm{mt}}$ in the intestine through the release of neuropeptides, serotonin, and/or Wnt ligands (Berendzen et al. 2016; Shao et al. 2016; Zhang et al. 2018).

Genetic screens for additional factors that activate the $\mathrm{UPR}^{\mathrm{mt}}$ have revealed important roles for mitochondrial ceramide produced by SPTL-1/serine palmitoyltransferase and sphingosine-1-phosphate (S1P) produced by SPHK-1/ sphingosine kinase in the activation of the UPR ${ }^{\mathrm{mt}}$ (Liu et al. 2014; Kim and Sieburth 2018a). SPHK-1 recruitment to mitochondria from cytoplasmic pools may serve as an early signal to activate UPR ${ }^{\mathrm{mt}}$ (Kim and Sieburth 2018a). SPHK-1 mitochondrial recruitment is positively regulated by mitochondrial stress originating either from the intestine or from the nervous system. Neuronal mitochondrial stress activates 
intestinal SPHK-1 by a mechanism that involves neuropeptide, but not serotonin, signaling (Kim and Sieburth 2018a). Neuropeptides exert their biological functions primarily through activating $\mathrm{G}$ protein-coupled receptors (GPCRs) on target cells to trigger downstream signaling events (Frooninckx et al. 2012). However, the specific neuropeptides and the GPCRs functioning in SPHK-1-mediated UPR ${ }^{\mathrm{mt}}$ activation have not been identified.

FSHR-1 is a GPCR-containing extracellular Leucine-Rich Repeats (LRRs) protein that is homologous to the folliclestimulating hormone receptor family (Powell et al. 2009). FSHR-1 plays a critical role in activating innate immunity in response to infection by pathogenic bacteria, and functions in the intestine to promote protection against pathogenic infection and to regulate antimicrobial gene expression. (Cho et al. 2007; Powell et al. 2009; Miller et al. 2015). Interestingly, infection by pathogenic bacteria leads to multiple cellular responses in the intestine, including the activation of the UPR $^{m t}$ (Liu et al. 2014; Pellegrino et al. 2014).

In this study, through the analysis of $f_{s h r-1}$ null mutants, we show that FSHR-1 positively regulates UPR ${ }^{\mathrm{mt}}$ activation and promotes the mitochondrial association of SPHK-1 in the intestine. Through tissue-specific rescue experiments, we find that FSHR-1 functions in the nervous system to exert its function in UPR ${ }^{\mathrm{mt}}$ activation. We propose that FSHR-1 is part of a neuroendocrine signaling network that functions to regulate the UPR ${ }^{\mathrm{mt}}$ through intertissue signaling.

\section{Materials and Methods}

\section{C. elegans strains}

Strains used in this study were maintained at $22^{\circ}$ following standard methods. Young adult hermaphrodites derived from the wild-type reference strain N2 Bristol were used for all experiments. The following mutant strains were used. SJ4100: zcIs13[Phsp-6::GFP], OJ4113: vjIs138[Pges-1:: sphk-1::gfp], OJ4143: vjIs148[Pges-1::tomm-20::mCherry], ST66: ncIs17[Phsp-16.2::gfp], OJ2329: vjIs208[Pgst-4::gfp], uthIs375[unc-119p::cco-1 HP + rol-6(su1006)](Zhang et al. 2018), OJ997: sphk-1(ok1097), GR2250: mgIs73[cyp-14A4p:: gfp], and KP3397: fshr-1(ok778). The sphk-1(ok1097) and $f_{s h r-1(o k 778)}$ strains were outcrossed at least six times with wild-type animals prior to analysis.

\section{Molecular biology}

fshr-1 complementary cDNA (cDNA) was cloned from C. elegans wild-type cDNA and then inserted into the pPD49.26 expression vector using standard molecular biology techniques. The following plasmids were generated: pSK55[Pges1::fshr-1], pSK56[Prab-3::fshr-1], and pSK98[Prgef-1::fshr-1]. Sequence files of plasmids are available upon request.

\section{Transgenic lines}

Transgenic strains were generated by injecting expression constructs (10-25 $\mathrm{ng} / \mu \mathrm{l})$ and the co-injection marker

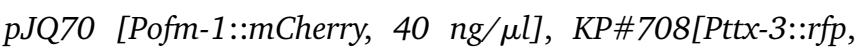
$40 \mathrm{ng} / \mu \mathrm{l}$ ], or KP\#1106 [Pmyo-2::gfp, $10 \mathrm{ng} / \mu \mathrm{l}]$ into N2 worms or the indicated mutants using standard techniques (Mello et al. 1991). At least three lines for each transgene were tested and a representative transgene was used for further experiments. The following transgenic lines were made: vjEx1448[Prab-3::fshr-1], vjEx1449[Pges-1::fshr-1], vjEx1000[Pges-1::gfp], and vjEx1831[Prgef-1::fshr-1].

\section{Microscopy and analysis}

Fluorescence microscopy experiments were performed following previous methods (Kim and Sieburth 2018a). Briefly, L4-stage or young adult worms were immobilized by using 2,3-butanedione monoxime (BDM, $30 \mathrm{mg} / \mathrm{ml}$; Sigma [Sigma Chemical], St. Louis, MO) in M9 buffer then mounted on 2\% agarose pads for imaging. To quantify the fluorescence intensity of Phsp-6::GFP or Pgst-4::GFP, Z stacks of the intestine posterior to the vulva were selected as a representative region because of low basal expression in the absence of stress. Images were captured with the Nikon (Garden City, NY) eclipse 90i microscope equipped with a Nikon PlanApo $40 \times$, or $60 \times$ or $100 \times$ objective (NA $=1.4$ ), and a PhotometricsCoolsnap ES2 or a Hamamatsu Orca Flash LT + CMOS camera. Metamorph 7.0 software (Universal Imaging/ Molecular Devices) was used to capture serial image stacks, and the maximum intensity was measured (Kim and Sieburth 2018 b). Intensity quantification analysis was performed on the same day to equalize the absolute fluorescence levels between samples within the same experimental set. For Figure $1 \mathrm{~A}$, each sample cluster of worms was captured using a $4 \times$ objective lens for the representative images as shown in Figure 1A, then captured by a $40 \times$ objective lens for fluorescence intensity quantification.

\section{RNA interference}

A feeding RNA interference (RNAi) knockdown assay was performed following established protocols (Kamath and Ahringer 2003). Briefly, gravid adult animals were placed on RNAi plates seeded with HT115(DE3) bacteria transformed with $\mathrm{L} 4440$ vector containing a genomic fragment of the gene to be knocked down (or empty L4440 vector as a control) to collect eggs, then removed after $4 \mathrm{hr}$ to obtain an age-matched synchronized worm population. Young adult animals were used for subsequent assays.

\section{Stress induction assays}

For drug-induced stress, transgenic L4 animals were transferred to fresh NGM plates seeded with HB101 bacteria, then $80 \mu \mathrm{l}$ of stock solutions dissolved in M9 buffer of paraquat were added to plates for a final concentration of $0.4 \mathrm{mM}$ paraquat. After $24 \mathrm{hr}$, adults were selected for fluorescence microscopy analysis. For imaging of animals that had been subjected to RNAi-induced knockdown, L4 animals grown on RNAi plates were transferred to new RNAi plates to obtain synchronized animals and imaged 24 hr later. For Pgst-4::GFP imaging, young adult animals were incubated with $5 \mathrm{mM}$ 

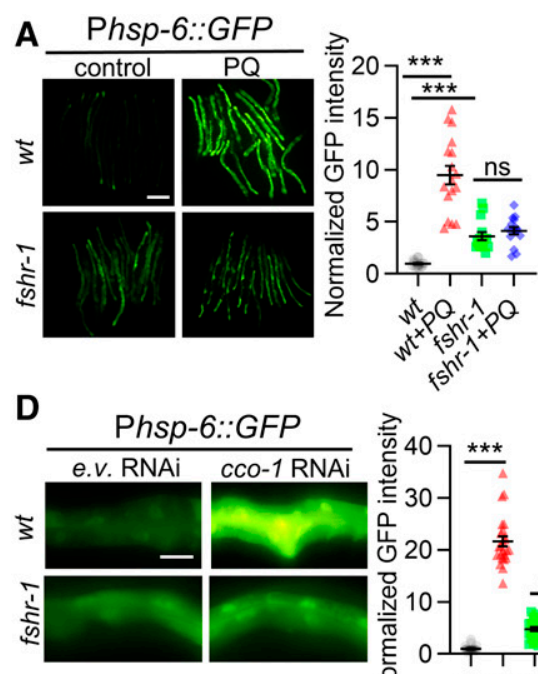

B
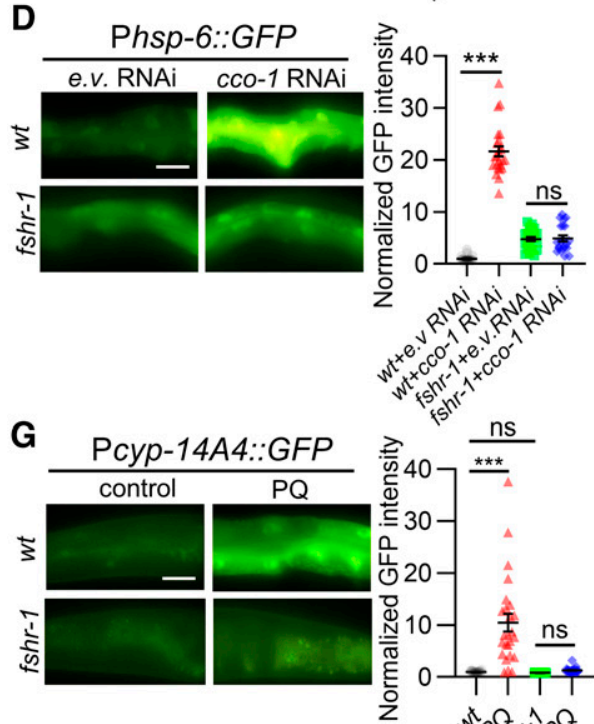

से ns
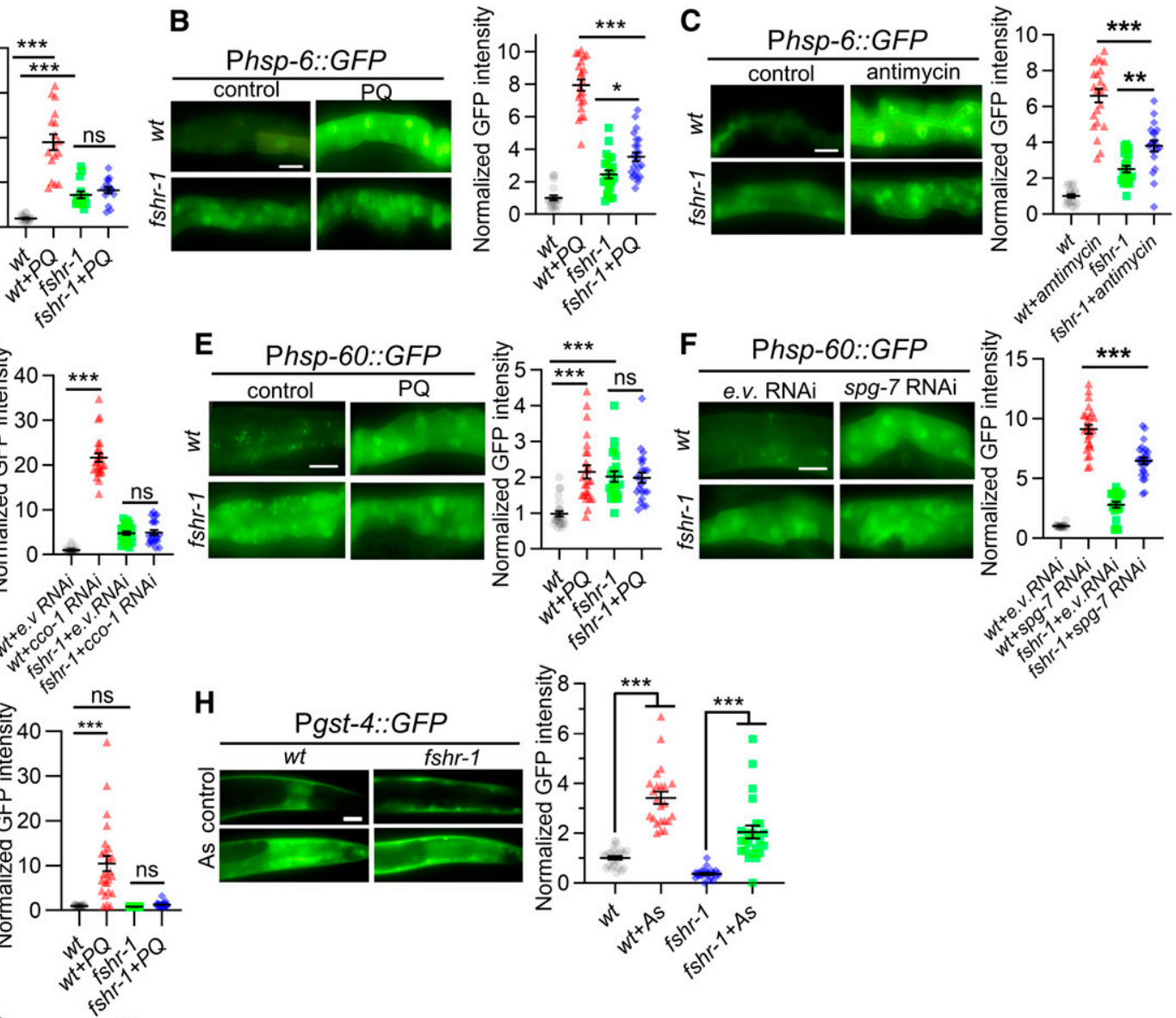

$\mathbf{F}$
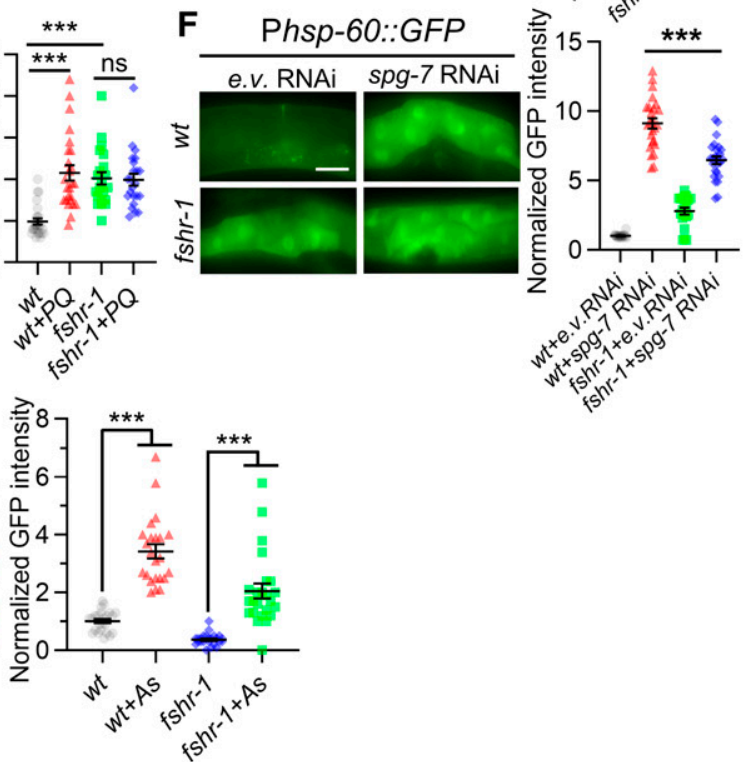
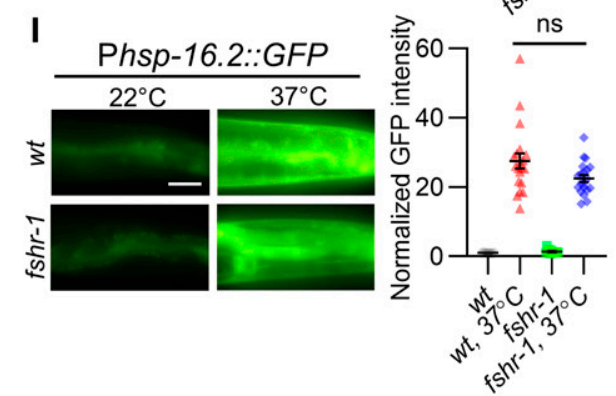

Figure 1 FSHR-1 promotes UPRmt activation. (A) Representative low-magnification images of several wt adults or fshr-1 mutants expressing the zcls 13 [Phsp-6::GFP] transgene (GFP driven by the hsp-6 promoter), following treatment with M9 (control) or PQ for 24 hr. (B and C) Left: representative highmagnification images of intestines posterior to the vulva in young adult wt animals or fshr-1 mutants expressing Phsp-6::GFP following treatment with M9 (control) or PQ (B), or DMSO (control) or antimycin (C) for $24 \mathrm{hr}$. Right: average GFP fluorescence intensity is quantified. (D) Left: representative images of Phsp-6::GFP fluorescence in intestines of wt or fshr-1 mutants following e.v. control or cco-1/cox-5B RNAi treatment by feeding. Right: average GFP fluorescence intensity is quantified. (E and F) Left: representative intestine images of wt or fshr-1 mutants expressing the zcls9 [Phsp60::GFP] transgene following M9 and e.v. (G) (control), or PQ or spg-7/AFG3L2 RNAi treatment. Right: average GFP fluorescence intensity is quantified. (G) Left: representative images of wt or fshr-1 mutants expressing the mgls73 [Pcyp-14A4::gfp] transgene in the absence or presence of PQ. Right: average GFP fluorescence intensity is quantified. $(\mathrm{H})$ Left: representative images of posterior intestines from animals expressing the antioxidant reporter transgene [Pgst-4::GFP] in wt or fshr-1 mutants in the absence or presence of As. Right: average GFP fluorescence intensity is quantified. (I) Left: representative images of posterior intestines from animals expressing heat shock transgene ncls17 [Phsp-16.2::GFP] in wt or fshr-1 mutants following $22^{\circ}$ (control) or $37^{\circ}$ exposure for 20 min. Right: average GFP fluorescence intensity is quantified. Bar represents $10 \mu \mathrm{m}$. Error bars indicate \pm SEM. Oneway ANOVA with Bonferroni post-test $* P<0.1 ; * * P<0.01 ; * * * P<0.001$. As, arsenite; e.v., empty vector; ns, not statistically significant; $P Q$, paraquat; RNAi, RNA interference; UPR ${ }^{m t}$, mitochondrial unfolded protein response; wt, wild-type.

arsenite or $50 \mathrm{mM}$ paraquat in liquid solution (in M9 buffer) for $1 \mathrm{hr}$, then drug was washed out and worms were transferred onto new NGM plates for $4 \mathrm{hr}$ before imaging. M9 buffer was used as a control for paraquat and arsenite treatment.
For Phsp-16.2::GFP imaging, young adult animals were incubated at $22^{\circ}$ or $37^{\circ}$ for $20 \mathrm{~min}$, and then allowed to recover at $22^{\circ}$ for $24 \mathrm{hr}$ prior to imaging. For paraquat survival assays, young adult animals were placed onto NGM plates 
containing $10 \mathrm{mM}$ paraquat for $17 \mathrm{hr}$. After $17 \mathrm{hr}$, the percentage of surviving animals was counted every $3 \mathrm{hr}$ over the course of $15 \mathrm{hr}$. Survival assays were done in experimental duplicate for each biological duplicate.

\section{Statistical analysis}

We utilized one-way ANOVA with a Bonferroni post-test to evaluate the statistical significance for multiple sample comparisons including Figure 3B (26 hr), while Student's t-tests were used for two-sample comparison using Graphpad Prizm 8. $P$-values $<0.01$ or 0.001 are indicated with asterisks $(* * P<0.01$ and $* * * P<0.001$, respectively). Error bars in the figures indicate the SE of the mean ( \pm SEM). At least 15 animals per sample were analyzed for fluorescence quantification and data points, and averages are presented as shaped box plots in the figures.

\section{Data availability}

Strains and plasmids used in this study are available upon request. The authors confirm that all data necessary for confirming the conclusions of the findings are present within the article and figures.

\section{Results}

\section{FSHR-1/GPCR signaling regulates the UPR}

Prior studies found that $f s h r-1$ mutants have normal life spans (Powell et al. 2009), but exhibit enhanced sensitivity to lethality caused by the mitochondrial toxin and UPR ${ }^{\mathrm{mt}}$ activator paraquat (Miller et al. 2015). Because impairing UPR ${ }^{\mathrm{mt}}$ activation causes sensitivity to paraquat-induced lethality (Nargund et al. 2012; Gatsi et al. 2014; Liu et al. 2014; Kim and Sieburth 2018a), we speculated that FSHR-1 may positively regulate the $\mathrm{UPR}^{\mathrm{mt}}$. To monitor $\mathrm{UPR}^{\mathrm{mt}}$ activation, we quantified the intestinal fluorescence of the UPR ${ }^{\mathrm{mt}}$ transcriptional reporter $z$ cIs 13 , in which GFP is expressed under control of the $h s p-6$ promoter (Phsp-6::GFP). Paraquat is an oxidant that interferes with electron transport at the inner mitochondrial membrane, and has been used widely to acutely activate the UPR ${ }^{\mathrm{mt}}$ in both $C$. elegans and in mammalian cells (Nargund et al. 2012; Runkel et al. 2013; Fiorese et al. 2016; Kim and Sieburth 2018a). Wild-type animals treated with paraquat for $24 \mathrm{hr}$ exhibited a roughly 10-fold increase in Phsp-6::GFP expression in the intestine compared to nontreated controls (Figure 1, A and B). In contrast, fshr-1 mutants showed a slight increase in baseline Phsp-6::GFP expression, and either no significant change or a small increase in Phsp-6::GFP expression following paraquat treatment (Figure 1, A and B). Antimycin is a mitochondrial electron transport chain (ETC) stressor, and antimycin treatment for $24 \mathrm{hr}$ robustly induces the UPR ${ }^{\mathrm{mt}}$ (Liu et al. 2014). fshr-1 mutations attenuated the antimycin-induced increase in Phsp-6::GFP expression (Figure 1C). cco-1 (aka cox-5B) encodes the cytochrome $c$ oxidase subunit in complex IV, which is the terminal electron acceptor of the ETC, and
RNAi-mediated cco-1 knockdown is a potent activator of the UPR ${ }^{\mathrm{mt}}$ (Nargund et al. 2012; Berendzen et al. 2016; Merkwirth et al. 2016; Tian et al. 2016; Kim and Sieburth 2018a). cco-1 RNAi by feeding, which induces mitochondrial stress in the intestine (Zhang et al. 2018), significantly increased Phsp-6::GFP expression in wild-type animals but failed to increase Phsp-6::GFP expression in $f s h r-1$ mutants (Figure 1D). fshr-1 mutations also blocked or attenuated the stress-induced increase in expression of two additional UPR $^{\mathrm{mt}}$ reporters: Phsp-60::GFP and Pcyp-14A4::GFP [Figure 1, E-G, Nargund et al. (2012), and Mao et al. (2019)].

fshr-1 mutants were not defective in the induction of the antioxidant reporter Pgst-4::GFP in response to treatment with the mitochondrial reactive oxygen species (ROS) generator arsenite [Figure $1 \mathrm{H} ; 1.6$-fold increase in wild-type and 1.9-fold increase in $f s h r-1$ mutants by arsenite (Inoue et al. 2005; Choe et al. 2009; Ruiz-Ramos et al. 2009; Prakash et al. 2015; Wu et al. 2016)]. In addition, $f s h r-1$ mutants exhibited normal induction of the heat-shock reporter Phsp-16.2::GFP in response to heat shock at $37^{\circ}$ for 20 min (Figure 1I). Together, these results suggest that FSHR-1 plays a specific role in activating the $\mathrm{UPR}^{\mathrm{mt}}$ in response to mitochondrial stress.

\section{FSHR-1/GPCR functions cell nonautonomously in neurons to regulate UPR ${ }^{m t}$}

FSHR- 1 is expressed primarily in the intestine and in a subset of neurons (Sieburth et al. 2005; Cho et al. 2007; Cao et al. 2017). To determine in which tissue FSHR-1 functions to regulate the $\mathrm{UPR}^{\mathrm{mt}}$, we examined Phsp-6::GFP induction in transgenic $f_{s h r-1}$ mutants expressing $f_{s h r}-1$ cDNA in either the nervous system (using the $r a b-3$ or the rgef- 1 promoter) or the intestine (using the ges-1 promoter). Pan-neuronal fshr-1 either partially ( $r a b-3$ promoter) or fully (rgef-1 promoter) rescued the paraquat-induced $h s p-6$ expression defects of $f s h r-1$ mutants (Figure 2, A and B). In contrast, intestinal $f s h r-1$ cDNA expression failed to rescue the paraquat-induced $h s p-6$ expression defects of $f$ shr- 1 mutants (Figure $2 \mathrm{~A})$. RNAi-mediated knockdown of $f_{s h} \mathrm{r}-1$ by bacterial feeding, which efficiently knocks down genes in the intestine but not in the nervous system (Kamath et al. 2001; Timmons et al. 2001; Asikainen et al. 2005), did not attenuate paraquatinduced $h s p-6$ expression, confirming that $f$ shr- 1 does not function in the intestine to regulate the UPR ${ }^{\mathrm{mt}}$ (Figure 2D). Taken together, these results suggest that FSHR-1 primarily functions cell nonautonomously in the nervous system to positively regulate $\mathrm{UPR}^{\mathrm{mt}}$ activation.

The intestinal UPR ${ }^{\mathrm{mt}}$ can be activated by cell-autonomous stress originating in the intestine or by cell-nonautonomous stress originating in the nervous system (Nargund et al. 2012; Berendzen et al. 2016; Merkwirth et al. 2016; Zhang et al. 2018). fshr-1 is required for proper UPR ${ }^{\mathrm{mt}}$ activation elicited by intestinal stress (Figure 1, D and F). To determine whether $f s h r-1$ promotes $\mathrm{UPR}^{\mathrm{mt}}$ activation elicited by neuronal stress, we examined Phsp-6::GFP expression in animals in which $c c 0-1$ was selectively silenced in the nervous system. Neuronal cco-1 knockdown 

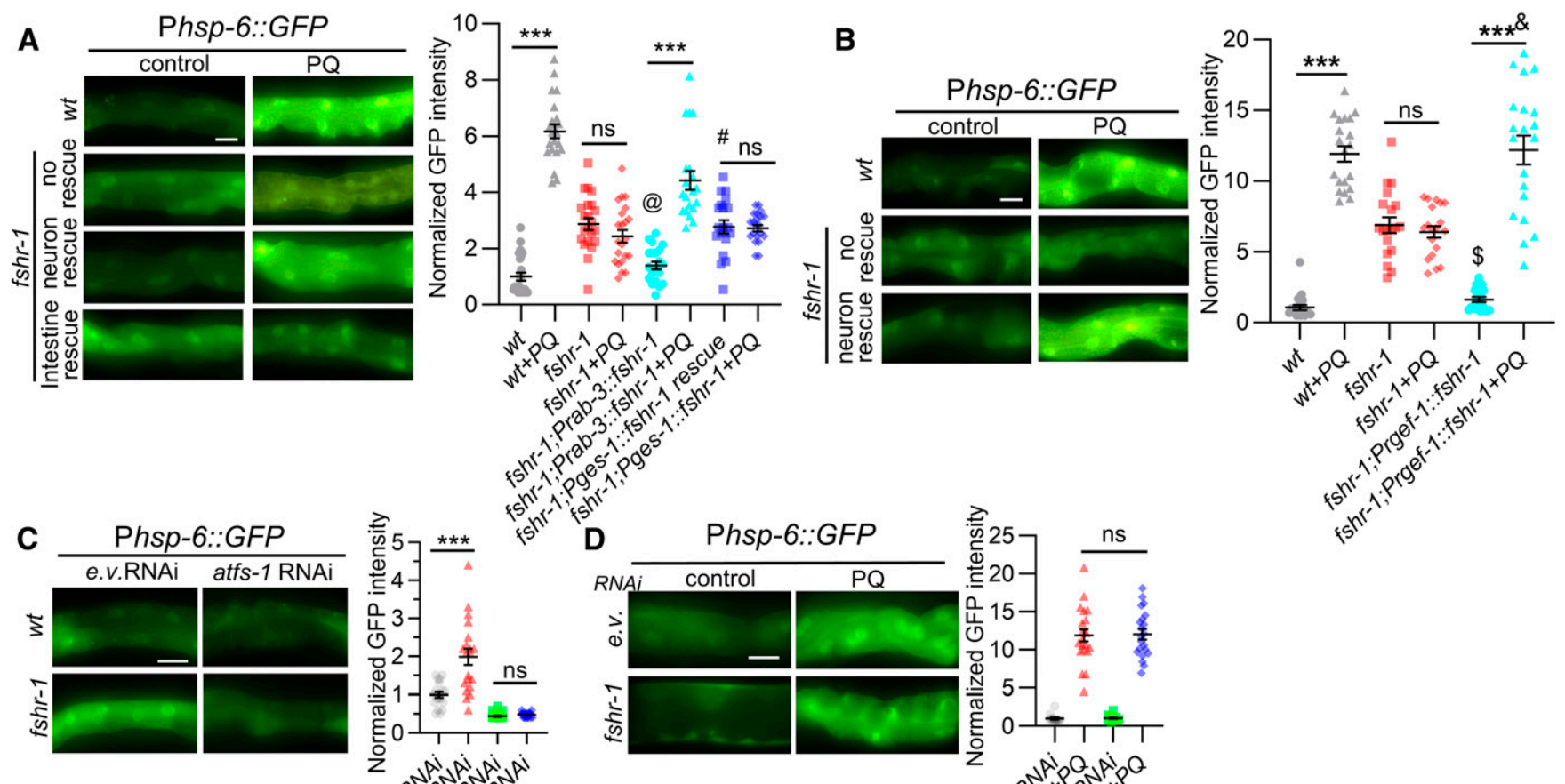

5.
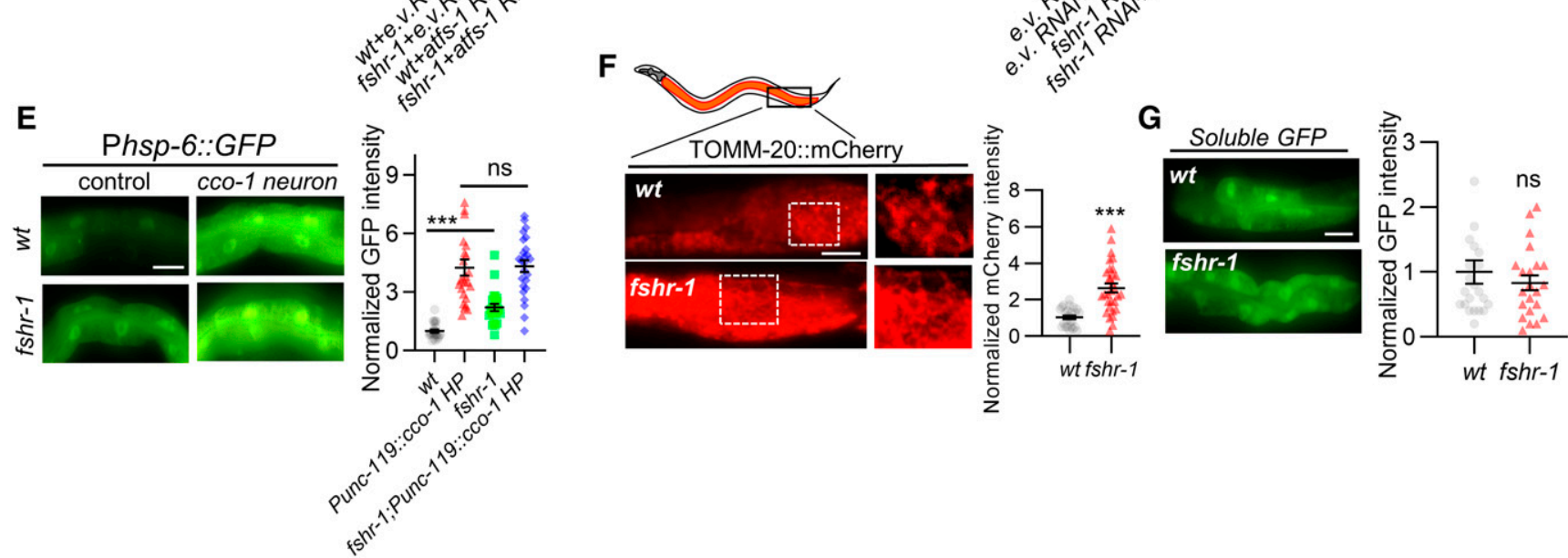

Figure 2 FSHR-1 functions in the nervous system to regulate the UPR ${ }^{m t}$. (A and B) Left: representative images of posterior intestines of young adult animals or fshr-1 mutants expressing the Phsp-6::GFP transgene following treatment with M9 (control) or PQ for 24 hr. "fshr-1 neuronal rescue" denotes fshr-1 mutants expressing fshr-1 cDNA under the neuron-specific promoter rab-3 (A) or rgef-1 (B). "fshr-1 intestine rescue" denotes fshr-1 mutants expressing fshr-1 CDNA under the intestine-specific promoter ges-1. Right: average GFP fluorescence intensity is quantified. (C) Left: representative images of intestines of wt or fshr-1 mutants expressing Phsp-6::GFP following e.v. control or atfs-1 RNAi treatment by feeding. Right: average GFP fluorescence intensity is quantified. (D) Left: representative images of intestines of wt worms expressing Phsp-6::GFP following e.v. control or fshr-1 RNAi treatment by feeding in the absence or presence of PQ. Right: average GFP fluorescence intensity is quantified. (E) Left: representative images of Phsp-6::GFP fluorescence in intestines of wt or fshr-1 mutants in the absence or presence of transgene uth/s375[unc-119p::cco-1 HP + rol-6(su1006)]]). Right: average GFP fluorescence intensity is quantified. (F) Left: representative images of intestinal cells of wt or fshr-1 mutants expressing the mitochondrial marker TOMM-20::mCherry under the intestinal-specific promoter ges-1. Right: average mCherry fluorescence intensities are quantified. (G) Left: representative images of animals expressing soluble GFP in the intestine (Pges-1::GFP) in wt or fshr-1 mutants. Right: average GFP fluorescence intensities are quantified. Bar in the panel represents $10 \mu \mathrm{m}$. Error bars indicate \pm SEM. One-way ANOVA with Bonferroni post-test $* * * P<0.001 ; \# P<$ 0.001 compared to fshr-1; Prab-3::fshr-1; @P>0.05 compared to wt; $\$ P>0.05$ compared to wt; and \&P>0.05 compared to wt + PQ. cDNA,

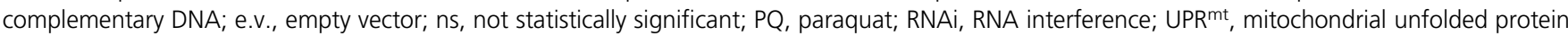
response; wt, wild-type.

by hairpin-mediated RNAi is a potent activator of the intestinal $\mathrm{UPR}^{\mathrm{mt}}$ (Zhang et al. 2018). Neuronal cco-1 knockdown elicited similar intestinal Phsp-6::GFP expression in $f_{s h}$ - 1 mutants as in wild-type controls (Figure $2 \mathrm{E}$ ). The fold induction was slightly reduced in $f_{s h} r-1$ mutants [4.3-fold for $f s h r-1(+)$ vs. 2.2 fold for $f s h r-1(-)$ ] due to increased baseline $h s p-6$ expression in $f s h r-1$ mutants (Figure $2 \mathrm{E})$. Thus, $f_{s h} r-1$ appears to be largely dispensable for $\mathrm{UPR}^{\mathrm{mt}}$ activation when stress originates from the nervous system. 


\section{Neuronal FSHR-1 regulates baseline hsp-6 and hsp-60 expression}

Under nonstressed conditions, fshr-1 mutants exhibited small but significant increases in Phsp-6::GFP expression in the intestine compared to wild-type controls (Figure 1, A-D), and larger increases in Phsp-60::GFP expression (Figure 1, E and F) that could reach levels similar to those seen under stressed conditions (Figure 1E). The increase in Phsp-6::GFP expression was blocked by RNAi-mediated knockdown of atfs-1 (Figure 2C) and was restored to wild-type levels by pan-neuronal, but not by intestinal, $f_{s h} r-1$ cDNA expression (Figure 2, A and B), suggesting that the UPR ${ }^{\mathrm{mt}}$ is activated even in the absence of stress in $f$ shr- 1 mutants. Together, these results indicate that neuronal FSHR-1 functions cell nonautonomously to keep $\mathrm{UPR}^{\mathrm{mt}}$ activity low in the absence of stress.

Mitochondria marked by the mitochondrial localization signal of TOMM-20 fused to mCherry (TOMM-20::mCherry) are distributed in a highly reticulated pattern within the cytoplasm of intestinal cells [expressed under the ges-1 promoter; Figure 2E, Palikaras et al. (2015), and Kim and Sieburth (2018a)]. fshr-1 mutants exhibited a normal overall pattern of TOMM-20:mCherry fluorescence in intestinal cells (Figure 2E), but the average fluorescence intensity of TOMM-20:mCherry associated with mitochondria was significantly increased by about threefold in $f$ shr-1 mutants compared to wild-type controls (Figure 2F). In control experiments, we found that $f s h r-1$ mutants exhibited no change in the fluorescence intensity of soluble GFP expressed under the ges-1 promoter (Figure 2G), suggesting that transgene expression was not altered in $f$ shr-1 mutants. The increase in TOMM-20 fluorescence intensity in $f$ sh $r-1$ mutants could reflect either an increase in mitochondrial mass, a decrease in TOMM-20 turnover, or an increase in TOMM-20 targeting to the outer mitochondrial membrane. Alterations in mitochondrial mass, morphology, or mitochondrial protein import have been linked to UPR ${ }^{\mathrm{mt}}$ activation (Ungvari et al. 2011; Houtkooper et al. 2013; Lerner et al. 2013; Mouchiroud et al. 2013; Mao et al. 2019).

\section{FSHR-1 and SPHK-1 function in a common pathway to activate the UPR ${ }^{m t}$}

SPHK-1 functions in the intestine to activate the UPR in response to a variety of mitochondrial stressors, including paraquat (Kim and Sieburth 2018a). To test whether FSHR-1 functions in a common pathway with SPHK-1 to activate the UPR ${ }^{\mathrm{mt}}$, we examined genetic interactions between sphk-1 and fshr-1 mutants. sphk-1 mutants exhibited a significant reduction in paraquat-induced expression of Phsp$6:: G F P$ ( $89 \%$ reduction), which is similar to that exhibited by $f$ sh -1 mutants [92\% reduction; Figure 3A and Kim and Sieburth (2018a)]. Double mutants lacking both $s p h k-1$ and $f s h r-1$ exhibited increased baseline expression of $h s p-6$, which was similar to that of $f$ sh $r-1$ mutants, and had defects in paraquat-induced $h s p-6$ induction that were no more severe than those seen in single mutants (93\% reduction, Figure 3A). sphk-1 mutants exhibit reduced survival rates when exposed to toxic levels of paraquat (Kim and Sieburth 2018a), which are similar to those of $f$ shr- 1 mutants (Figure $3 \mathrm{~B}$ ). The survival rates of double mutants lacking both $s p h k-1$ and $f s h r-1$ were no more severe than those of either single mutant (Figure 3B). Expression of $s p h k-1$ cDNA in the intestine fully rescued the increased paraquat-induced lethality of $s p h k-1$ mutants (Kim and Sieburth 2018a). Expression of $f_{s h r-1}$ cDNA in the nervous system fully rescued the increased paraquat-induced lethality of $f s h r-1$ mutants, whereas expression of $f s h r-1$ cDNA in the intestine partially restored paraquat sensitivity to fshr-1 mutants [Figure 3B and Miller et al. (2015). Taken together, FSHR-1 and SPHK-1 may function in a common pathway to activate the UPR ${ }^{\mathrm{mt}}$, and to promote organism-wide protection from mitochondrial stress-induced lethality. Moreover, FSHR-1 functions in the nervous system to activate the UPR ${ }^{\mathrm{mt}}$ and promote survival, whereas SPHK-1 functions exclusively in the intestine.

\section{FSHR-1/GPCR regulates mitochondrial association of $S P H K-1$ in the intestine}

SPHK-1 localizes to intestinal mitochondria and is recruited to mitochondria from cytosolic pools by mitochondrial stress (Kim and Sieburth 2018a). SPHK-1::GFP fusion proteins, which are fully functional in rescuing the $U P R^{m t}$ defects of sphk-1 mutants, colocalize with TOMM-20::mCherry (Figure 4A). Mitochondrial stress induces a significant increase in mitochondrial SPHK-1::GFP fluorescence, as well as an increase in the number of intestinal cells in which SPHK1::GFP adopts a mitochondrial distribution pattern [referred to as mitochondrial occupancy; Figure $4 \mathrm{~B}$ and Kim and Sieburth (2018a)]. To determine whether FSHR-1 regulates SPHK-1 mitochondrial association, we examined the mitochondrial abundance of SPHK-1::GFP fusion proteins before and after paraquat exposure. fshr-1 mutants exhibited two defects in SPHK-1::GFP mitochondrial association. First, $f_{s h r-1}$ mutants exhibited a nearly twofold reduction in SPHK-1::GFP mitochondrial association compared to wildtype controls in the absence of paraquat (Figure 4B). Second, the paraquat-induced increase of mitochondrial SPHK$1:$ GFP association was completely abolished in $f s h r-1$ mutants (Figure 4B). Thus, FSHR-1 positively regulates SPHK-1 association with mitochondria under nonstressed conditions and FSHR-1 is required for stress-induced SPHK-1 mitochondrial targeting.

\section{Neuronal fshr-1 cell-nonautonomously regulates mitochondrial association of SPHK-1 in the intestine}

To determine in which tissue FSHR-1 functions to regulate the mitochondrial association of SPHK-1, we quantified SPHK-1::GFP mitochondrial fluorescence in $f$ shr-1 mutants expressing $f s h r-1$ cDNA in either the nervous system or in the intestine. We found that pan-neuronal expression of

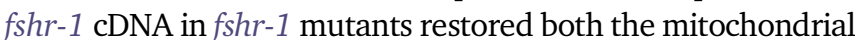
occupancy and fluorescence intensity of SPHK-1::GFP to wildtype levels in the absence of stress. Similarly, neuronal $f_{s h r}-1$ 

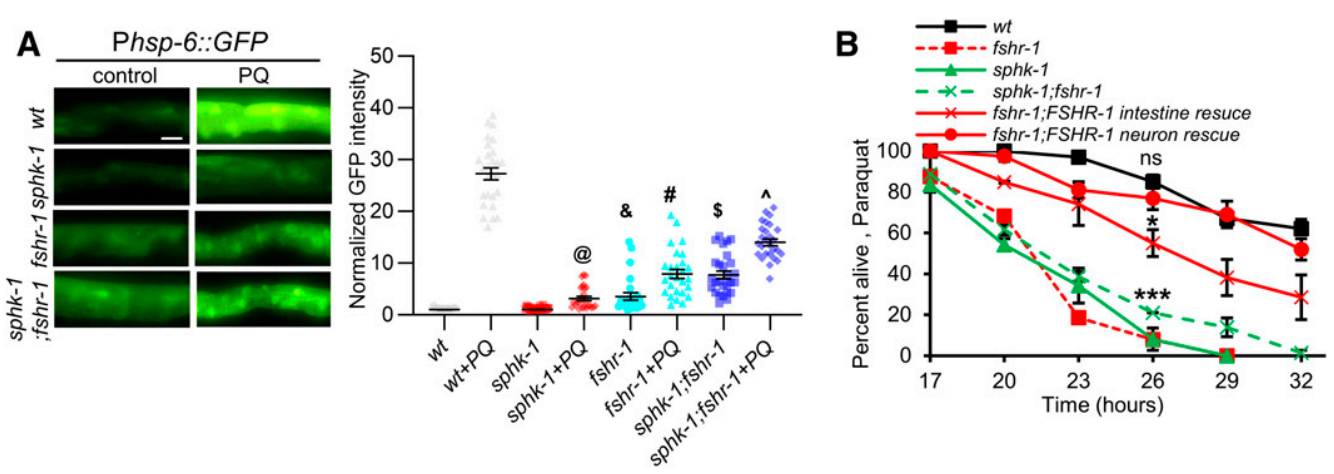

Figure 3 FSHR-1 functions in a common pathway with SPHK-1 to activate the UPR't. (A) Left: representative images of Phsp-6::GFP expression in the intestines of wild-type, sphk-1 mutants, fshr-1 mutants, and sphk-1; fshr-1 double mutants in the absence or the presence of $\mathrm{PQ}$ for $24 \mathrm{hr}$. Right: average GFP fluorescence intensity is quantified. (B) Survival rate curves of the indicated strains on plates containing $10 \mathrm{mM} \mathrm{PQ}$. Bar represents $10 \mu \mathrm{m}$. One-way ANOVA with Bonferroni post-test: @P $<0.001$ compared to sphk-1; \&P<0.001 compared to sphk-1; \#P<0.001 compared to fshr-1; $\$ P<0.001$ compared to fshr-1; and $\wedge P<0.001$ compared to sphk-1;fshr-1; ns: $P>0.05$ compared to fshr-1; FSHR-1 neuron rescue; *P<0.05 compared to wt; and $* * * P<$ 0.001 compared to wt. ns, not statistically significant; PQ, paraquat; UPR ${ }^{m t}$, mitochondrial unfolded protein response; wt, wild-type.

cDNA also restored normal paraquat-induced SPHK-1::GFP mitochondrial recruitment to $f_{s h}$ - 1 mutants. In contrast, intestinal fshr-1 cDNA expression failed to rescue the SPHK$1::$ GFP fluorescence defects of $f s h r-1$ mutants (Figure 4B). These results suggest that FSHR-1 functions exclusively in the nervous system to positively regulate baseline SPHK-1 abundance in the intestine in the absence of stress, and to promote paraquat-induced SPHK-1 mitochondrial association.

\section{Discussion}

The nervous system coordinates various stress responses by releasing diffusible factors that act upon distal tissues to activate cellular defense programs (Nussbaum-Krammer and Morimoto 2014; Berendzen et al. 2016; Shao et al. 2016). Here, we show that the conserved GPCR FSHR-1 is part of an integrated organism-wide response to mitochondrial stress that functions to activate the UPR ${ }^{\mathrm{mt}}$ in the intestine. $f_{s h r}-1$ promotes $\mathrm{UPR}^{\mathrm{mt}}$ activation in response to either acute exposure to mitochondrial toxins or to chronic mitochondrial dysfunction, and functions in a common pathway with sphk-1 to promote survival in response to toxic mitochondrial stress and UPR ${ }^{\mathrm{mt}}$ activation. $f_{s} h r-1$ positively regulates the mitochondrial association of SPHK-1 in the intestine in the absence of stress, as well as stress-induced SPHK-1 mitochondrial recruitment. $f_{s h}$ - 1 functions in the nervous system, but not in the intestine, to promote $\mathrm{UPR}^{\mathrm{mt}}$ activation and stress-induced SPHK-1 mitochondrial recruitment. Finally, neuronal $f_{s h r}-1$ regulates intestinal mitochondrial homeostasis in the absence of stress by a mechanism that may involve regulating mitochondrial mass and/or mitochondrial protein abundance. We propose a model whereby neuronal $f s h r-1$ signaling keeps UPR ${ }^{\mathrm{mt}}$ activity low in the absence of stress and positively regulates the UPR ${ }^{\mathrm{mt}}$ in response to intestinal stress. The underlying mechanism by which $f s h r-1$ regulates the UPR ${ }^{\mathrm{mt}}$ may be by establishing or maintaining proper mitochondrial homeostasis in the intestine.

fshr-1 functions cell nonautonomously in the nervous system to activate the UPR ${ }^{\mathrm{mt}}$; however, our genetic evidence suggests that it is not likely to function in one of the previously identified "mitokine" pathways that signal neuronal stress to the intestine. Disruption of these pathways (e.g., egl-20/Wnt or flp-2/neuropeptide) blocks the ability of stress originating in the nervous system to promote intestinal $\mathrm{UPR}^{\mathrm{mt}}$ activation (Shao et al. 2016; Kim and Ewbank 2018; Zhang et al. 2018). We found that $f s h r-1$ is required for UPR ${ }^{\mathrm{mt}}$ activation in response to intestinal stress but not neuronal stress. Thus, $f_{s h}-1$ is not likely to be a component of a neuronal mitokine pathway but instead may promote UPR ${ }^{\mathrm{mt}}$ activation by a mechanism that does not involve the direct response to neuronal stress.

Previous studies have established an important role for FSHR-1 in activating the innate immune response following infection with pathogenic bacteria. FSHR-1 promotes both survival following infection and the behavioral avoidance response to pathogenic bacteria, and FSHR-1 activates a number of antimicrobial and antioxidant genes in response to pathogens (Powell et al. 2009; Miller et al. 2015). FSHR-1 is proposed to not participate in the detection of the pathogenic bacteria themselves, but instead to participate in the detection of cellular damage (e.g., ROS) resulting from bacterial infection (Miller et al. 2015). Pathogenic bacterial infection is also a potent activator of the UPR ${ }^{\mathrm{mt}}$, and UPR ${ }^{\mathrm{mt}}$ activation by pathogens promotes survival by inducing the expression of innate immune genes (Pellegrino et al. 2014). We speculate that $f s h r-1$ may activate the innate immune response by contributing to activation of the UPR ${ }^{\mathrm{mt}}$ in response to a broad array of insults (e.g., pathogenic infection, ROS, and mitochondrial dysfunction). Interestingly, we found that intestinal $f_{s h r}-1$ does not regulate the UPR ${ }^{\mathrm{mt}}$, yet intestinal $f s h r-1$ expression protects $f s h r-1$ mutants from paraquat toxicity, suggesting that activation of the UPR ${ }^{\mathrm{mt}}$ may not be necessary to elicit resistance to paraquat. Paraquat is an ROS generator that also activates the intestinal antioxidant response, and $f s h r-1$ promotes the expression of antioxidant genes (Powell et al. 2009; Miller et al. 2015). Thus, the paraquat sensitivity of $f_{s h} r-1$ mutants may arise from defects in the antioxidant response in the intestine.

FSHR-1 also regulates intestinal mitochondrial homeostasis in the absence of stress. Whether FSHR-1 does so by 


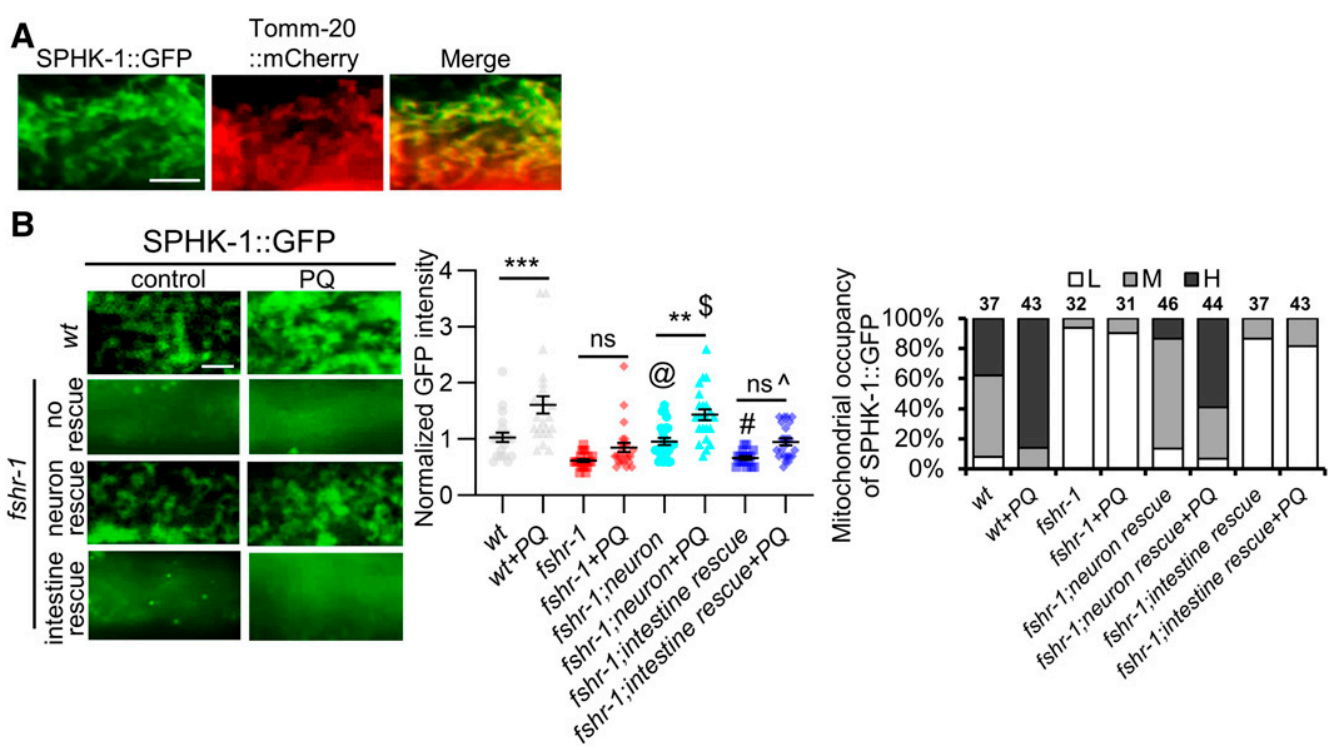

Figure 4 FSHR-1 regulates mitochondrial association of SPHK-1. (A) High-magnification images of SPHK-1::GFP (left), TOMM-20::GFP (middle), and merge (right) in wt animals coexpressing Pges-1::sphk1::gfp and Pges-1::tomm-20::mCherry transgenes. (B) Left: representative images of the indicated mutants expressing intestinal SPHK-1::GFP in the absence or presence of $\mathrm{PQ}$ for $24 \mathrm{hr}$. fshr-1 neuron and intestine rescue denote transgenic fshr-1 mutants expressing fshr-1 cDNA under the pan-neural promoter rab-3 or the intestinal promoter ges-1, respectively. Middle: average fluorescence intensities in the indicated strains expressing SPHK-1::GFP are quantified. Right: mitochondrial occupancy of indicated transgenes is quantified in the absence or presence of PQ. $\mathrm{H}, \mathrm{M}$, or L indicates the percentage of animals showing mitochondrially localized SPHK-1::GFP in more than two-thirds of the intestine $(\mathrm{H})$, one-half of the intestine $(\mathrm{M})$, or only in a few intestinal cells $(\mathrm{L})$. Bar represents $2 \mu \mathrm{m}$. One-way ANOVA with Bonferroni post-test $* * P<$ $0.01 ; * * * P<0.001 ; @ P>0.05$ compared to wt; $\# P>0.05$ compared to fshr-1 mutants; $\$ P>0.05$ compared to wt + $P Q ;$ and $\wedge P>0.05$ compared to fshr-1 + PQ. cDNA, complementary DNA; ns, not statistically significant; PQ, paraquat; wt, wild-type.

regulating mitochondrial mass or mitochondrial protein import, or both, is not clear based on our quantitative imaging of mitochondrial markers. Interestingly, FSHR signaling negatively regulates mitochondrial biogenesis in mammals (Liu et al. 2017). Enhanced mitochondrial biogenesis is correlated with $\mathrm{UPR}^{\mathrm{mt}}$ activation. Inducers of mitochondrial biogenesis, such as nicotinamide riboside and poly(ADP-ribose) polymerase inhibitors (PARPi), disrupt mito-nuclear protein homeostasis resulting in $\mathrm{UPR}^{\mathrm{mt}}$ activation via the sirtuin 1 (SIRT1) pathway in mammals and C. elegans. (Mouchiroud et al. 2013). In addition, rapamycin and resveratrol, which increase mitochondrial content, also induce $\mathrm{UPR}^{\mathrm{mt}}$ activation in C. elegans (Ungvari et al. 2011; Houtkooper et al. 2013; Lerner et al. 2013). In worms, mutants with defects in the import of mitochondrial proteins into the matrix exhibit increased $h s p-60$ expression that is dependent upon atfs-1 (Nargund et al. 2012). We speculate that the defects in paraquat-induced UPR ${ }^{\mathrm{mt}}$ activation in $f$ shr-1 mutants may be due to an underlying defect in the mitochondrial association of SPHK-1 under normal conditions.

Since FSHR-1 functions to protect animals from diverse stressors, the ligand for FSHR-1 is likely to originate from the host rather than from an exogenous source (Powell et al. 2009). FSHR-1 shares homology with three human GPCRs-FSHR, TSHR, and LHCGR - by virtue of nine LRRs found in its extracellular domain (Dolan et al. 2007). These GPCRs are activated by the heterodimeric glycopeptide hormones $\mathrm{FSH} \alpha / \beta$, TSH $\alpha / \beta$, and $\mathrm{LH} \alpha / \beta$, respectively. In humans, FSH induces the generation of S1P by stimulating SphK1 activity for granulosa cell proliferation (Hernández-Coronado et al. 2016), suggesting potential functional conservation of ligand-activated FSHR-1 signaling. An additional mammalian glycopeptide hormone family member, thyrostimulin, is a heterodimer composed of the GPB5 $\alpha$-subunit and the GPA2 $\beta$-subunit, which share homology with worm T23B12.8 and FLR-2, respectively (Rocco and Paluzzi 2016). Interestingly, genetic analysis implicates flr-2 in the neuronal control of intestinal functions (Oishi et al. 2009), but whether flr-2 regulates the $\mathrm{UPR}^{\mathrm{mt}}$ or innate immunity has not been investigated. Identifying the ligand(s) for FSHR-1 will help to clarify the cellnonautonomous mechanisms by which FSHR-1 regulates the UPR ${ }^{\mathrm{mt}}$ pathway.

\section{Acknowledgments}

We thank the Ruvkun laboratory for providing the mgIs73 reporter strain and the Dillin laboratory for providing the uthIs375 strain. This work was supported by grants from the National Institutes of Health (NIH) National Institute of Neurological Disorders and Stroke to D.S. (NS-071085 and NS-099414). Some strains were provided by the Caenorhabditis Genetics Center, which is funded by the NIH Office of Research Infrastructure Programs (P40 OD-010440).

\section{Literature Cited}

Asikainen, S., S. Vartiainen, M. Lakso, R. Nass, and G. Wong, 2005 Selective sensitivity of Caenorhabditis elegans neurons to RNA interference. Neuroreport 16: 1995-1999 [corrigenda: Neuroreport 17: 230-231 (2006)]. https://doi.org/10.1097/ 00001756-200512190-00005

Berendzen, K. M., J. Durieux, L. W. Shao, Y. Tian, H. E. Kim et al., 2016 Neuroendocrine coordination of mitochondrial stress signaling and proteostasis. Cell 166: 1553-1563.e10. https:// doi.org/10.1016/j.cell.2016.08.042 
Cao, J., J. S. Packer, V. Ramani, D. A. Cusanovich, C. Huynh et al., 2017 Comprehensive single-cell transcriptional profiling of a multicellular organism. Science 357: 661-667. https://doi.org/ 10.1126/science.aam8940

Cho, S., K. W. Rogers, and D. S. Fay, 2007 The C. elegans glycopeptide hormone receptor ortholog, FSHR-1, regulates germline differentiation and survival. Curr. Biol. 17: 203-212. https:// doi.org/10.1016/j.cub.2006.12.027

Choe, K. P., A. J. Przybysz, and K. Strange, 2009 The WD40 repeat protein WDR-23 functions with the CUL4/DDB1 ubiquitin ligase to regulate nuclear abundance and activity of SKN-1 in Caenorhabditis elegans. Mol. Cell. Biol. 29: 2704-2715. https:// doi.org/10.1128/MCB.01811-08

Dolan, J., K. Walshe, S. Alsbury, K. Hokamp, S. O'Keeffe et al., 2007 The extracellular leucine-rich repeat superfamily; a comparative survey and analysis of evolutionary relationships and expression patterns. BMC Genomics 8: 320 [corrigenda: BMC Genomics 10: 230 (2009)]. https://doi.org/10.1186/1471-2164-8-320

Durieux, J., S. Wolff, and A. Dillin, 2011 The cell-non-autonomous nature of electron transport chain-mediated longevity. Cell 144: 79-91. https://doi.org/10.1016/j.cell.2010.12.016

Fiorese, C. J., A. M. Schulz, Y. F. Lin, N. Rosin, M. W. Pellegrino et al., 2016 The transcription factor ATF5 mediates a mammalian mitochondrial UPR. Curr. Biol. 26: 2037-2043. https:// doi.org/10.1016/j.cub.2016.06.002

Frooninckx, L., L. Van Rompay, L. Temmerman, E. Van Sinay, I. Beets et al., 2012 Neuropeptide GPCRs in C. elegans. Front. Endocrinol. (Lausanne) 3: 167. https://doi.org/10.3389/ fendo.2012.00167

Gatsi, R., B. Schulze, M. J. Rodriguez-Palero, B. Hernando-Rodriguez, R. Baumeister et al., 2014 Prohibitin-mediated lifespan and mitochondrial stress implicate SGK-1, insulin/IGF and mTORC2 in C. elegans. PLoS One 9: e107671. https://doi.org/10.1371/ journal.pone.0107671

Hernández-Coronado, C. G., A. Guzmán, A. Rodríguez, J. A. Mondragón, M. C. Romano et al., 2016 Sphingosine-1-phosphate, regulated by FSH and VEGF, stimulates granulosa cell proliferation. Gen. Comp. Endocrinol. 236: 1-8. https://doi.org/10.1016/ j.ygcen.2016.06.029

Houtkooper, R. H., L. Mouchiroud, D. Ryu, N. Moullan, E. Katsyuba et al., 2013 Mitonuclear protein imbalance as a conserved longevity mechanism. Nature 497: 451-457. https://doi.org/ 10.1038 /nature 12188

Inoue, H., N. Hisamoto, J. H. An, R. P. Oliveira, E. Nishida et al., 2005 The C. elegans p38 MAPK pathway regulates nuclear localization of the transcription factor SKN-1 in oxidative stress response. Genes Dev. 19: 2278-2283. https://doi.org/10.1101/ gad.1324805

Kamath, R. S., and J. Ahringer, 2003 Genome-wide RNAi screening in Caenorhabditis elegans. Methods 30: 313-321. https:// doi.org/10.1016/S1046-2023(03)00050-1

Kamath, R. S., M. Martinez-Campos, P. Zipperlen, A. G. Fraser and J. Ahringer, 2001 Effectiveness of specific RNA-mediated interference through ingested double-stranded RNA in Caenorhabditis elegans. Genome Biol. 2: RESEARCH0002. https:// doi.org/10.1186/gb-2000-2-1-research0002

Kim, D. H., and J. J. Ewbank, 2018 Signaling in the innate immune response (August 14, 2018), WormBook, ed. The C. elegans Research Community WormBook, doi/10.1895/wormbook.1.83.2, http://www.wormbook.org. https://doi.org/10.1895/wormbook. 1.83 .2

Kim, S., and D. Sieburth, 2018a Sphingosine kinase activates the mitochondrial unfolded protein response and is targeted to mitochondria by stress. Cell Rep. 24: 2932-2945.e4. https:// doi.org/10.1016/j.celrep.2018.08.037

Kim, S., and D. Sieburth, 2018b Sphingosine kinase regulates neuropeptide secretion during the oxidative stress-response through intertissue signaling. J. Neurosci. 38: 8160-8176. https://doi.org/10.1523/JNEUROSCI.0536-18.2018

Lerner, C., A. Bitto, D. Pulliam, T. Nacarelli, M. Konigsberg et al., 2013 Reduced mammalian target of rapamycin activity facilitates mitochondrial retrograde signaling and increases life span in normal human fibroblasts. Aging Cell 12: 966-977. https:// doi.org/10.1111/acel.12122

Liu, Y., B. S. Samuel, P. C. Breen, and G. Ruvkun, 2014 Caenorhabditis elegans pathways that surveil and defend mitochondria. Nature 508: 406-410. https://doi.org/10.1038/nature13204

Liu, P., Y. Ji, T. Yuen, E. Rendina-Ruedy, V. E. DeMambro et al., 2017 Blocking FSH induces thermogenic adipose tissue and reduces body fat. Nature 546: 107-112. https://doi.org/10.1038/ nature 22342

Mao, K., F. Ji, P. Breen, A. Sewell, M. Han et al., 2019 Mitochondrial dysfunction in C. elegans activates mitochondrial relocalization and nuclear hormone receptor-dependent detoxification genes. Cell Metab. 29: 1182-1191.e4. https://doi.org/ 10.1016/j.cmet.2019.01.022

Martinez, B. A., D. A. Petersen, A. L. Gaeta, S. P. Stanley, G. A. Caldwell et al., 2017 Dysregulation of the mitochondrial unfolded protein response induces non-apoptotic dopaminergic neurodegeneration in C. elegans models of Parkinson's disease. J. Neurosci. 37: 11085-11100. https://doi.org/10.1523/ JNEUROSCI.1294-17.2017

Mello, C. C., J. M. Kramer, D. Stinchcomb, and V. Ambros, 1991 Efficient gene transfer in C.elegans: extrachromosomal maintenance and integration of transforming sequences. EMBO J. 10: 3959-3970. https://doi.org/10.1002/j.1460-2075.1991. tb04966.x

Merkwirth, C., V. Jovaisaite, J. Durieux, O. Matilainen, S. D. Jordan et al., 2016 Two conserved histone demethylases regulate mitochondrial stress-induced longevity. Cell 165: 1209-1223. https://doi.org/10.1016/j.cell.2016.04.012

Miller, E. V., L. N. Grandi, J. A. Giannini, J. D. Robinson, and J. R. Powell, 2015 The conserved G-protein coupled receptor FSHR-1 regulates protective host responses to infection and oxidative stress. PLoS One 10: e0137403. https://doi.org/ 10.1371/journal.pone.0137403

Mouchiroud, L., R. H. Houtkooper, N. Moullan, E. Katsyuba, D. Ryu et al., 2013 The $\mathrm{NAD}(+) /$ Sirtuin pathway modulates longevity through activation of mitochondrial UPR and FOXO signaling. Cell 154: 430-441. https://doi.org/10.1016/j.cell.2013.06.016

Nargund, A. M., M. W. Pellegrino, C. J. Fiorese, B. M. Baker, and C. M. Haynes, 2012 Mitochondrial import efficiency of ATFS-1 regulates mitochondrial UPR activation. Science 337: 587590. https://doi.org/10.1126/science.1223560

Nargund, A. M., C. J. Fiorese, M. W. Pellegrino, P. Deng, and C. M. Haynes, 2015 Mitochondrial and nuclear accumulation of the transcription factor ATFS-1 promotes OXPHOS recovery during the UPR(mt). Mol. Cell 58: 123-133. https://doi.org/10.1016/ j.molcel.2015.02.008

Nussbaum-Krammer, C. I., and R. I. Morimoto, 2014 Caenorhabditis elegans as a model system for studying non-cell-autonomous mechanisms in protein-misfolding diseases. Dis. Model. Mech. 7: 31-39. https://doi.org/10.1242/dmm.013011

Oishi, A., K. Gengyo-Ando, S. Mitani, A. Mohri-Shiomi, K. D. Kimura et al., 2009 FLR-2, the glycoprotein hormone alpha subunit, is involved in the neural control of intestinal functions in Caenorhabditis elegans. Genes Cells 14: 1141-1154. https:// doi.org/10.1111/j.1365-2443.2009.01341.x

Palikaras, K., E. Lionaki, and N. Tavernarakis, 2015 Coordination of mitophagy and mitochondrial biogenesis during ageing in C. elegans. Nature 521: 525-528. https://doi.org/10.1038/ nature 14300

Pellegrino, M. W., A. M. Nargund, N. V. Kirienko, R. Gillis, C. J. Fiorese et al., 2014 Mitochondrial UPR-regulated innate 
immunity provides resistance to pathogen infection. Nature 516: 414-417. https://doi.org/10.1038/nature13818

Powell, J. R., D. H. Kim, and F. M. Ausubel, 2009 The G proteincoupled receptor FSHR-1 is required for the Caenorhabditis elegans innate immune response. Proc. Natl. Acad. Sci. USA 106: 2782-2787. https://doi.org/10.1073/pnas.0813048106

Prakash, C., M. Soni, and V. Kumar, 2015 Biochemical and molecular alterations following arsenic-induced oxidative stress and mitochondrial dysfunction in rat brain. Biol. Trace Elem. Res. 167: 121-129. https://doi.org/10.1007/s12011-015-0284-9

Rocco, D. A., and J. P. Paluzzi, 2016 Functional role of the heterodimeric glycoprotein hormone, GPA2/GPB5, and its receptor, LGR1: an invertebrate perspective. Gen. Comp. Endocrinol. 234: 20-27. https://doi.org/10.1016/j.ygcen.2015.12.011

Ruiz-Ramos, R., L. Lopez-Carrillo, A. D. Rios-Perez, A. De VizcayaRuiz, and M. E. Cebrian, 2009 Sodium arsenite induces ROS generation, DNA oxidative damage, HO-1 and c-Myc proteins, NF-kappaB activation and cell proliferation in human breast cancer MCF-7 cells. Mutat. Res. 674: 109-115. https://doi.org/ 10.1016/j.mrgentox.2008.09.021

Runkel, E. D., S. Liu, R. Baumeister, and E. Schulze, 2013 Surveillanceactivated defenses block the ROS-induced mitochondrial unfolded protein response. PLoS Genet. 9: e1003346 [corrigenda: PLoS Genet. 12: e1006377 (2016)]. https://doi.org/10.1371/journal. pgen.1003346

Shao, L. W., R. Niu, and Y. Liu, 2016 Neuropeptide signals cell non-autonomous mitochondrial unfolded protein response. Cell Res. 26: 1182-1196. https://doi.org/10.1038/cr.2016.118
Sieburth, D., Q. Ch'ng, M. Dybbs, M. Tavazoie, S. Kennedy et al., 2005 Systematic analysis of genes required for synapse structure and function. Nature 436: 510-517. https://doi.org/10. 1038/nature03809

Tian, Y., G. Garcia, Q. Bian, K. K. Steffen, L. Joe et al., 2016 Mitochondrial stress induces chromatin reorganization to promote longevity and UPR(mt). Cell 165: 1197-1208. https://doi.org/10.1016/j.cell.2016.04.011

Timmons, L., D. L. Court, and A. Fire, 2001 Ingestion of bacterially expressed dsRNAs can produce specific and potent genetic interference in Caenorhabditis elegans. Gene 263: 103-112. https://doi.org/10.1016/S0378-1119(00)00579-5

Ungvari, Z., W. E. Sonntag, R. de Cabo, J. A. Baur, and A. Csiszar, 2011 Mitochondrial protection by resveratrol. Exerc. Sport Sci. Rev. 39: 128-132. https://doi.org/10.1097/ JES.0b013e3182141f80

Wu, C. W., A. Deonarine, A. Przybysz, K. Strange, and K. P. Choe, 2016 The Skp1 homologs SKR-1/2 are required for the Caenorhabditis elegans SKN-1 antioxidant/detoxification response independently of p38 MAPK. PLoS Genet. 12: e1006361. https://doi.org/10.1371/journal.pgen.1006361

Zhang, Q., X. Wu, P. Chen, L. Liu, N. Xin et al., 2018 The mitochondrial unfolded protein response is mediated cellnon-autonomously by retromer-dependent Wnt signaling. Cell 174: 870-883 e7. https://doi.org/10.1016/j.cell.2018. 06.029

Communicating editor: B. Grant 